\title{
Plataforma colaborativa para auxílio na identifica- ção de casos suspeitos de doenças da Tríplice epi- demia e focos do mosquito Aedes Aegypit
}

Title: Collaborative platform to aid in the identification of suspected cases of diseases of the triple epidemic and outbreaks of the Aedes Aegypit mosquito

\author{
André Luiz Ximenes Gonçalves da Luz \\ Escola Politécnica de Pernambuco \\ Universidade de Pernambuco \\ 50.720-001 - Recife, Brasil \\ andreluizximenes@gmail.com
}

\author{
Danilo Ricardo Barbosa de Araújo \\ Escola Politécnica de Pernambuco \\ Universidade de Pernambuco \\ 50.720-001 - Recife, Brasil \\ danilo.araujo@ufrpe.br
}

\begin{abstract}
Resumo Dentre as tecnologias aplicadas à vigilância em saúde, o mapeamento de epidemias se destaca pela importância em detectar possiveis pontos de surtos de uma determinada doença e com isso possibilitar uma alocação de recursos mais eficiente para aquela localidade. Neste sentido, a proposta deste trabalho é o desenvolvimento de uma plataforma Web que possa detectar casos suspeitos de doenças da Tríplice Epidemia e possíveis focos de proliferação do mosquito Aedes Aegypit. Com estas informações poderá ser feita uma relação entre a quantidades de casos relatados e os possíveis focos situados em uma determinada localidade, indicando áreas que necessitem de uma atenção especial por parte dos órgãos responsáveis e da sociedade em geral. Para tanto, desenvolveu-se o PortalVCS com o uso de conceitos como o design responsivo e tecnologias como Web Services, JavaScript e Firebase. O sistema foi avaliado por 32 usuários em potencial e as opiniões sobre o sistema foram positivas, pois segundo $90 \%$ usuários eles usariam o sistema novamente. A partir dos testes e das análises realizadas percebeu-se a importância de uma ferramenta colaborativa que seja um aliado no combate ao mosquito e as doenças causadas por ele.
\end{abstract}

Palavras-Chave: Vigilância Colaborativa, Vigilância em Saúde, Tecnologia da Informação e comunicação, Epidemia, Surto, Mapeamento, Dengue, Zika, Chikungunya, Geolocalização

\begin{abstract}
Among the technologies applied to health surveillance, mapping epidemics highlights the importance to detect possible points of outbreaks of some disease and thus enable a more efficient resource allocation for that location. In this sense, the purpose of this work was the development of a Web platform that can detect suspected cases of diseases of the Triple epidemic and possible sources of proliferation of Aedes Aegypit. With this information it could be possible to make a link between the amounts of reported cases and possible outbreaks located in a particular locality, indicating areas that require special attention from the responsible agent and society in general. Thus, the PortalVCS was developed with the use of concepts like responsive design and technologies such as Web Services and JavaScript Firebase. The system was evaluated by 32 potential users and their opinions on the system were positive. According to $90 \%$ of them, they would use the system again. From the tests and the analyzes we realized the importance of a collaborative tool that works as an ally in the fight against the mosquitoes and the diseases caused by it.
\end{abstract}

Keywords: Collaborative Surveillance, Health Surveillance, Communication and Information Technology, Epidemic, Outbreak, Mapping, Dengue, Zika, Chikungunya, Geolocation 


\section{Introdução}

Nos últimos vinte anos, os estudos epidemiológicos utilizados na vigilância em saúde adotaram novas metodologias e novas ferramentas graças as mudanças feitas no Regulamento Sanitário Internacional (RSI), cuja versão lançada em 2005 permitiu o uso de fontes não oficiais para detecção precoce de surtos e epidemias. A partir de então, além de considerar as notificações e consultas oficiais, os estudos passaram a contar com o uso de informações disponíveis na mídia em geral e na Internet, a exemplo dos websites, plataformas e ferramentas colaborativas, em geral denominadas de crowdsourcing (GOMES, 2012; LEAL-NETO OB et al, 2016).

Dessa forma, o uso das Tecnologias da Informação e Comunicação (TIC) na saúde vem crescendo e se consolidando a cada ano, tendo como objetivo principal: melhorar o fluxo de informações, monitoramento e prestação de serviços através dos meios eletrônicos. Este uso específico das TIC na área da saúde é conhecido como eHealth ou e-Health (VISCONTI, 2015).

Dentre as tecnologias aplicadas à vigilância em saúde, o mapeamento de epidemias se destaca pela importância em detectar possíveis pontos de surtos de uma determinada doença e com isso possibilitar uma alocação de recursos mais eficiente para aquela localidade (VISCONTI, 2015).

De acordo como o boletim epidemiológico (2016) volume $47 \mathrm{~N}^{\circ} 20$ do Ministério da Saúde, o estado registrou um total de 51.527 casos de dengue, 13.285 casos de chikungunya e 367 casos de zika (MINISTÉRIO DA SAÚDE, 2016). Ao analisar o histórico da Tríplice epidemia em Pernambuco é possível perceber que no cenário atual o Estado carece de ações que possam facilitar a detecção de possíveis focos do mosquito bem como aprimorar a atualização dos dados a respeito das pessoas contaminadas em prol do efetivo combate as estas doenças.

Atualmente existem várias plataformas eletrônicas que auxiliam na detecção precoce de surtos e epidemias como também no mapeamento destas doenças infecciosas. Essas plataformas disponibilizam para a população e serviços de saúde dados que proporcionam panoramas epidemiológicos ao redor do mundo.

Entretanto, embora exista esta diversidade de plataformas, não foi possível encontrar ferramentas que fossem capazes de monitorar ao mesmo tempo possíveis casos de doenças e as possíveis fontes de origem das mesmas. Um usuário pode está doente em casa, mas não necessariamente ter sido picado pelo mosquito lá, como também ele pode ter saído de casa para trabalhar, já doente, mas resolveu informar no sistema apenas quando estava no seu local de trabalho. A possibilidade do usuário ter a liberdade de informar o local onde ficou doente, ou onde viu um foco do mosquito Aedes Aegypit (popularmente conhecido como mosquito da dengue) é muito importante pois facilita o registro das informações e garante uma maior precisão da localização da informação.

Esta é, portanto, a proposta deste trabalho: possibilitar uma plataforma que possa detectar casos suspeitos de doenças da Tríplice epidemia e possíveis foco de proliferação do mosquito Aedes Aegypit.

Esta ferramenta permitirá que o usuário colabore informando como está se sentindo no momento, e onde é possível encontrar possíveis focos de proliferação do mosquito. Com estas informações poderá ser feita uma relação entre a quantidades de casos relatados e os possíveis focos situados em uma determinada localidade. Esta relação pode indicar áreas que necessitem de uma atenção especial por parte dos órgãos responsáveis e da sociedade em geral.

O restante deste artigo está organizado da seguinte forma: a Seção 2, denominada Trabalhos Relacionados, apresenta um breve histórico sobre os trabalhos que estão relacionados ao uso da tecnologia da informação e vigilância colaborativa para a detecção de epidemias. A Seção 3 engloba o referencial teórico e elenca as técnicas e tecnologias geralmente utilizadas no desenvolvimento de aplicações Web. A Seção 4 explica a metodologia utilizada no desenvolvimento de todas as etapas deste trabalho. A Seção 5 descreve a proposta do sistema, inclui uma explicação de todos os casos de uso dele e fala como e onde as tecnologias foram utilizadas no desenvolvimento do sistema. A Seção 6 mostra os resultados obtidos durante o período que de coleta de informações e por fim, na Seção 7 são feitas as considerações finais sobre todo o trabalho.

\section{Trabalhos Relacionados}

Dentre as plataformas identificadas no decorrer da elaboração deste projeto, foram selecionadas aquelas que estão diretamente relacionadas ao uso da tecnologia da informação e da vigilância colaborativa para a detecção de epidemias e que, consequentemente, serviram como base para o desenvolvimento da plataforma que será aqui proposta. Dessa forma, estão elencadas as seguintes plataformas: Promed, GPHIN, HealthMap, Observatório da Dengue, InfluenzaNet, Flu Near You, Dengue na Web e Guardiões da Saúde.

Em 1996, Morse SS, Rosenberg BH e Woodall J desenvolveram o ProMed, ferramenta que investigam a Web, coleta e reúne dados e dissemina de forma global informações sobre surto de doenças contagiosas através 
de um sistema automatizado de processamento de texto.

Já em 1997 foi desenvolvido o GPHIN, plataforma hoje mantida pelo governo canadense. Neste caso, o sistema coleta de forma contínua e sistemática por meio de palavras e frases-chaves dados da internet em sete idiomas (inglês, francês, espanhol, russo, árabe, chinês simplificado e tradicional). As informações são automaticamente separadas por relevância e analisadas por funcionários da agência canadense, após isso são disponibilizadas para os usuários.

Em 2008, após tentativas frustradas de obter dados em sites governamentais, Brownstein e Clark Freifeld criaram o HealthMap3. Uma plataforma que semelhante ao ProMed investiga a Internet procurando por relatos de doenças em sites, blogs, redes sociais, portais de notícias e documentos oficiais da Organização Mundial da Saúde (OMS). Essas informações são reunidas e disponibilizadas numa visão mais abrangente e unificada através de marcadores em um mapa. É possível assim acessar as fontes originais e também consumir as informações ali reunidas. O HealthMap visa atender em especial interesses de pessoas que trabalham com saúde pública e viajantes internacionais.

Em 2011, Barros et al desenvolveu o projeto Observatório da Dengue, o qual tinha como o objetivo o monitoramento de focos do mosquito transmissor desta doença. Este projeto funcionava em duas plataformas: a mobile (Android) e a Web. Os agentes de endemias da cidade utilizavam o aplicativo em um dispositivo mobile em suas visitas nas residências, pois o mesmo possuía um questionário onde era preenchido pelo próprio agente. $\mathrm{O}$ aplicativo tinha acesso a geolocalização atual do agente e incluía nos dados e eles eram submetidos para o servidor. Na Web os dados poderiam ser visualizados no mapa, onde cada visita aparecia usando a geolocalização capturada durante o preenchimento do questionário.

O InfluenzaNet é um sistema de monitoramento de casos de gripe do tipo (ILI) com a ajuda de voluntários através da internet. Teve seu início na Holanda e Bélgica em 2003 e devido ao seu sucesso foi expandida para outros países como Portugal (2005) e Itália (2008). Ao contrário do sistema tradicional de monitoramento de dengue, que é feito através de casos confirmados por médicos, o InfluenzaNet obtém seus dados diretamente da população, através de um questionário online. Qualquer residente de um país onde o InfluenzaNet está em funcionamento pode contribuir. Esses dados são analisados e processados e a partir daí é possível extrair informações cruciais que ajudam a combater possíveis surtos da gripe em uma determinada região.

O Flu Near You foi criado em 2011 nos Estados Uni-dos por epidemiologistas de Harvard, Boston Children's Hospital e o The Skoll Global Threats Fund. Tem ideia semelhante ao InfluenzaNet, no qual todos os dados obtidos para análise é proveniente de voluntários que preenchem um questionário online que é disponibilizado pela plataforma.

No Brasil, o projeto Dengue na Web surgiu no ano de 2011. Foi desenvolvido por um grupo de estudantes da UFBA (Universidade Federal da Bahia) e colaboração de pesquisadores do Instituto Gulbenkian de Ciência (IGC) de Portugal. Seu principal objetivo é coletar informações relacionadas aos sintomas da dengue com o intuito de identificar onde pode estar ocorrendo casos da doença na cidade de Salvador e com isso auxiliar o sistema de vigilância epidemiológica no combate a esta doença na capital da Bahia. Os dados são coletados através de um questionário disponível no seu website.

Em Pernambuco, a plataforma Guardiões da Saúde foi desenvolvida pela startup pernambucana Epitrack em parceria com o Ministério da Saúde e a Secretaria de Saúde das Sedes dos Jogos Olímpicos. Surgiu a partir do aperfeiçoamento de outra aplicação nomeada "Saúde na Copa" e que foi usada como teste no período da Copa do Mundo de 2014 no Brasil. Esta aplicação teste teve mais de 10 mil downloads e mais de 5 mil pessoas que utilizaram mais de uma vez. Através dos dados obtidos nesse período foi possível identificar vários conglomerados de doenças. Nesse sentido, o objetivo do Guardiões da Saúde foi conseguir identificar surtos da forma mais rápida possível, onde mesmo que o usuário não procure o sistema de saúde, seja possível identificar e se antecipar a possíveis epidemias ou surtos de doenças. $\mathrm{O}$ aplicativo tem uma verão mobile compatível com os sistemas Androide e IOS, mas também tem uma versão Web que é possível acessar de qualquer navegador.

\section{Referencial Teórico}

Nesta seção será falado um pouco sobre todos os conceitos de desenvolvimento Web e tecnologias utilizadas no desenvolvimento do trabalho proposto.

\subsection{Sistemas Web}

De modo geral, os sistemas Web são todos os sistemas projetados para serem utilizados em um navegador, como o Internet Explorer, Google Chrome, Mozilla Firefox, etc. Ficam hospedados em algum servidor de aplicações Web e pode ser acessada através de algum endereço (DE CASTRO).

Para o desenvolvimento de um sistema Web, quase sempre é utilizado padrões de desenvolvimento e linguagens, dentre as mais comuns estão: HTML (HyperText Markup Language), CSS (Cascade Style Sheet), JavaScript, PHP, Java, dentre outras. É comum dividir um sistema Web em duas partes: client-side (lado do cliente) 
e server-side (lado do servidor).

O cliente-side é a parte da aplicação responsável pela apresentação, interação com usuário e coleta dos dados. Nessa parte do sistema é utilizado padrões de desenvolvimento e linguagens como: HTML, CSS, JavaScript.

O server-side é responsável pela disponibilização de dados utilizados para a geração das páginas Web, é possível criar páginas dinâmicas on the fly para uma melhor experiência de uso. Também é onde todas as informações originadas no client-side são processadas, tratadas e armazenadas. Aqui geralmente são utilizadas linguagens como: PHP, Java, .Net, Python, JavaScript. É no serverside também que fica o banco de dados, onde serão armazenados todas as informações.

Normalmente a comunicação entre o client-side e server-side é feita usando o protocolo HTTP (Hypertext Transfer Protocol), um protocolo baseado em requisições e respostas que possui uma série de métodos (GET, POST, PUT, DELETE). Para entender melhor, quando se acessa um site de notícias e clica-se no link "esportes", o navegador, envia uma requisição HTTP para o servidor, este por sua vez recebe a requisição, processa e envia uma resposta que no caso é o conteúdo HTML da sessão de esportes do portal de notícias. Como resultado o usuário verá a página com as notícias sobre esportes. Toda resposta HTTP possui um cabeçalho e nele sempre vem o código de resposta que também e conhecido como status, no exemplo acima o status seria o 200, que significa que a requisição foi concluída com sucesso (DAL MORO, 2011).

\subsection{Sistemas Web Responsivos}

Com o surgimento dos dispositivos móveis como tablets e smartphones, veio também a necessidade de um novo padrão de design para atender os diferentes tamanhos de telas e suas resoluções. A ideia é de que ao invés de criar dois projetos de interface com o usuário separados, um para dispositivos móveis e outro para dispositivos convencionais, cria-se apenas um projeto de interface que vai se adaptar a qualquer tipo de dispositivo de forma dinâmica (BATURAY, 2013).

Aplicações responsivas são possíveis graças a evolução nas técnicas de Web Design, o uso de técnicas de design adaptativo, design fluído e técnicas de estruturação HTML e CSS. Como resultado o mesmo site se adapta a qualquer tela e resolução, uma imagem pode ser redimensionada dependendo do tamanho da tela que o usuário está acessando e com isso ele terá uma melhor experiência no uso do site. A Figura 1 mostra a ideia do design responsivo (BATURAY, 2013).

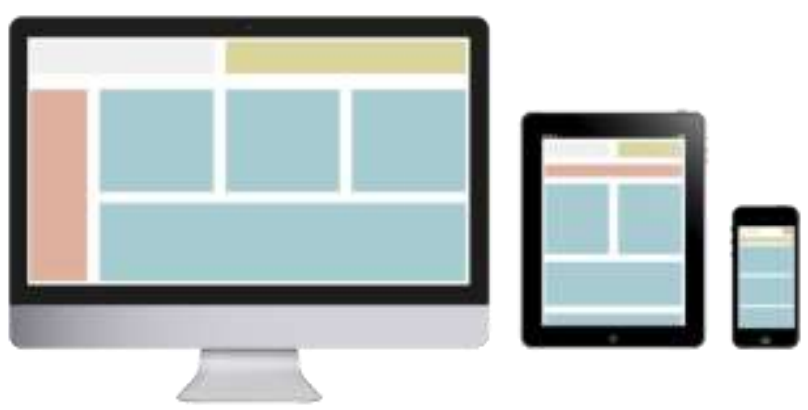

Figura 1: Uma mesma página em 3 dispositivos diferentes.

\subsection{Web Services}

De acordo com Hansen et. al. (Hansen et. al. 2002, Kreger 2001), Web Services são aplicações modulares, que podem ser descritas, publicadas e invocadas sobre uma rede, geralmente Web. Ou seja, é uma interface que descreve uma coleção de operações que são acessíveis pela rede através de mensagens em formato XML padronizadas. Permitem uma integração de serviços de maneira rápida e eficiente

Essa tecnologia permite que sistemas construídos em plataformas diferentes interajam entre si através de uma linguagem universal como o XML e o JSON.

Os Web Services que utilizam o padrão SOAP (Simple Object Access Protocol) trabalham com troca de mensagens XML que tem seu formato definido por um XML schema, seu principal objetivo é estabelecer um padrão para a comunicação de objetos e serviços. Basicamente é constituído de um elemento raíz que é o envelope, o qual identifica o arquivo XML como uma mensagem SOAP. Um elemento Header que possui informações do cabeçalho, um elemento Body responsável pelo conteúdo da mensagem e um elemento Fault responsável por manter informações e status sobre erros. Esse tipo de Web Services possui também o WSDL (Web Service Description Language) que tem como finalidade mostrar ao consumidor do serviço quais informações devem ser enviadas e o que será retornado por ele (DAL MORO, 2011)..

Os Web Services REST (Representational State Transfer), ao contrário do SOAP que tinha como objetivo estabelecer um padrão, tem como o objetivo de como utilizar corretamente os métodos do protocolo HTTP (GET, POST, PUT, DELETE, ETC) de forma semântica, ou seja, para busca de informações usar-se o GET, para atualização de informações usar-se o PUT, para salvar informações o POST e para excluir o DELETE. Seguindo esse padrão é possível utilizar uma única URI para manipular um determinado tipo de objeto. Como exemplo um serviço que pode ser definido apenas por uma URL http://servicoexemplo.com/usuario/123 , basta realizar uma requisição GET para este serviço que terei as infor- 
mações sobre o usuário de código 123, caso eu queira excluir é só fazer uma requisição DELETE para o mesmo endereço. Uma das vantagens do REST é que diferente do SOAP, o uso não se restringe apenas ao XML, é possível utilizar também o JSON (DAL MORO, 2011).

Outra característica importante no REST é o HATEOAS (Hypermedia As The Engine Of Application Status), considerado um dos principais benefícios dessa arquitetura, essa propriedade define que que todos os recursos de um Web Service devem possuir links que permitam o cliente saber quais ações são disponíveis a partir do ponto que está.

\subsection{Padrão MVC}

Model-View-Controller (MVC) é um padrão arquitetural que surgiu originalmente como um artefato idiomático na linguagem Smalltalk (Harrison et al. 2007). Com o seu uso, o sistema é dividido em três camadas: Model, View e Controller. A camada Model mantém as informações relacionadas ao domínio e objetos que implementam as funcionalidades de um sistema de informação.

$\mathrm{Na}$ camada View encontram-se os objetos relacionados à apresentação, ou seja, objetos que representam a interface gráfica com o usuário. A camada Controller define a maneira como a interface gráfica (View) deve agir, a partir das informações fornecidas pelo usuário, além de atualizar as informações e o estado dos objetos da camada Model. Assim, a camada Controller atua uma ponte, tendo acesso às classes da camada Model para realizar as tarefas do sistema, como por exemplo, registrar os dados de um cliente fornecidos através da interface gráfica. A realização dessa tarefa ocorre com a camada de interface gráfica com o usuário (View) coletando os dados do usuário e repassando-os para a camada Controller que, com os dados fornecidos, instancia um objeto da classe Cliente e persiste as informações desse objeto no banco de dados (Harrison et al. 2007).

\subsection{API}

Define-se que uma API (Application Programming Interface) ou interface de programação de aplicativos é um conjunto de rotinas e padrões estabelecidos por um software, para acesso a suas funcionalidades, sem a necessidade de envolver-se na implementação de tal.

Como exemplo para melhor entendimento, uma API muito conhecida e utilizada é a API de login do Facebook, a qual permite que aplicativos implementem o login do usuário com a sua conta do Facebook, eliminando assim a burocracia dos formulários de cadastro. A API de login do Facebook, fornece algumas informações públicas do usuário como o seu e-mail, nome, foto e id. Já a do Yahoo oferece clima e tempo, onde é possível obter informações sobre clima de um determinado local.

\section{Metodologia}

Para o desenvolvimento deste trabalho foi seguido cinco etapas para uma melhor definição da proposta final. $\mathrm{Na}$ primeira etapa, foi realizada a revisão da literatura técnica e científica a qual consistiu a buscas por trabalhos que abordassem os conceitos, tecnologias e trabalhos relacionados. A partir dela foi possível criar uma base de entendimento para um melhor refinamento da proposta. A revisão da literatura ficou dividida em duas partes, a primeira, voltada para trabalhos referentes ao problema que seria as epidemias, doenças causadas pelo mosquito da dengue, etc. E a segunda parte voltada para a busca por trabalhos e ferramentas desenvolvidos como uma possível solução para o problema. Nessa etapa também foram consultadas duas médicas do Hospital das Clínicas de Pernambuco, para um melhor entendimento sobre a tríplice epidemia, quais sintomas eram mais característicos, como era feito o trabalho de combate e registro de casos. Também foi consultado um profissional da área de combates a endemias da prefeitura de São Lourenço da Mata, onde pôde-se se entender melhor como funcionava o trabalho de busca a possíveis focos do mosquito da dengue. A consulta a esses profissionais foi importante pois a partir disso foi definido, como seriam as telas de registro de sintomas causados pelas doenças, e a tela de registro de focos do mosquito da dengue.

$\mathrm{Na}$ segunda etapa foi definida a arquitetura do sistema. Com a ajuda dos trabalhos relacionados, de conceitos de desenvolvimento e do conhecimento das tecnologias aplicadas nesse tipo de sistema, ficou definido que o sistema funcionaria no ambiente Web e usaria conceitos de design responsivo para que pudesse ser utilizado, tanto em de dispositivos convencionais, como em dispositivos móveis e faria o uso de uma API REST para a manipulação e armazenamento dos dados.

$\mathrm{Na}$ terceira etapa foi feita a implementação do sistema, iniciando por um protótipo navegacional, seguido da implementação e da validação do protótipo. Após a codificação, o sistema foi submetido a testes de usabilidade, e a partir dos resultados obtidos, ele sofreu algumas modificações a fim de melhorar a interação com o usuário.

$\mathrm{Na}$ quarta etapa o sistema foi disponibilizado para os usuários utilizarem. Foi divulgado em grupo de amigos, conhecidos e redes sociais. O sistema ficou disponibilizado por sete dias ( 25 de julho a 05 de agosto de 2016) para a coleta de informações referente a focos do mosquitos, diagnósticos e sintomas característicos das doenças.

$\mathrm{Na}$ quinta etapa foi realizada a análise dos resultados obtidos, na qual foi observada a quantidade de casos registrados por cidades e estes foram separados por tipos de informações, registros de focos do mosquito da dengue ou casos de sintomas e doenças. 


\section{Descrição da Plataforma}

Com o intuito de apresentar uma plataforma de vigilância participativa em saúde, foi desenvolvido o PortalVCS (Portal de Vigilância Colaborativa em Saúde) para o auxílio no monitoramento e detecção de possíveis surtos de doenças da tríplice epidemia, bem com na detecção de possíveis focos e criadouros do mosquito transmissor da dengue e também auxiliar o sistema de vigilância epidemiológica do estado de Pernambuco. A Figura 2 mostra uma visão geral da arquitetura do PorralVCS

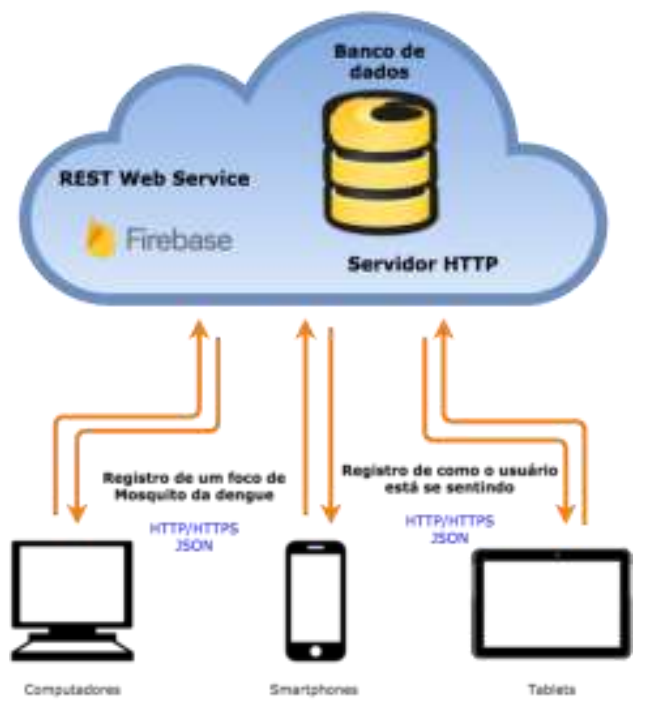

Figura 2: visão geral do PortalVCS.

\subsection{Casos de Uso}

No PortalVCS o usuário poderá informar como está se sentindo: se está se sentindo bem, ou se ele foi diagnosticado com uma das três doenças causadas pelo mosquito da dengue, bem como se está apresentando alguns dos sintomas característicos dessas doenças. Ele também terá a possibilidade de informar um possível foco de proliferação do mosquito da dengue como entulhos de lixo, casas ou prédios abandonados, etc. A Figura 3 ilustra os casos de uso da aplicação.

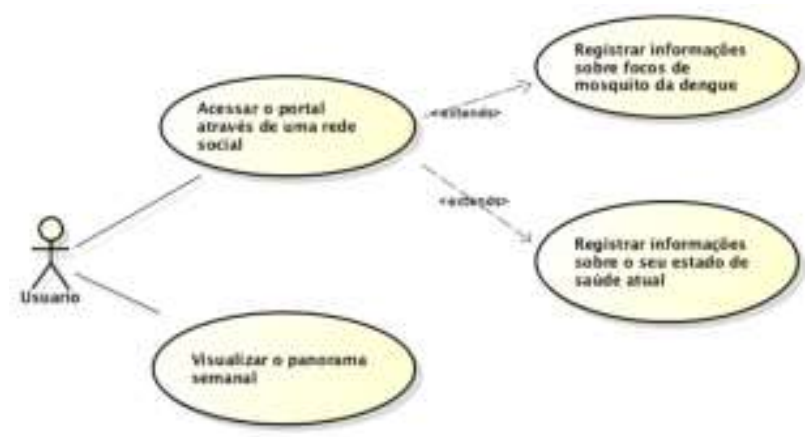

Figura 3: casos de usos da aplicação.
Para usar o PortalVCS o usuário acessará o endereço: https://portalcolaborativo.firebaseapp.com no navegador de sua preferência, bem como pelo seu smartphone ou tablet, então ele visualizará a pagina principal, com algumas instruções e dois botões "clique aqui para colaborar" e "visualizar panorama semanal", conforme apresentado na Figura 4

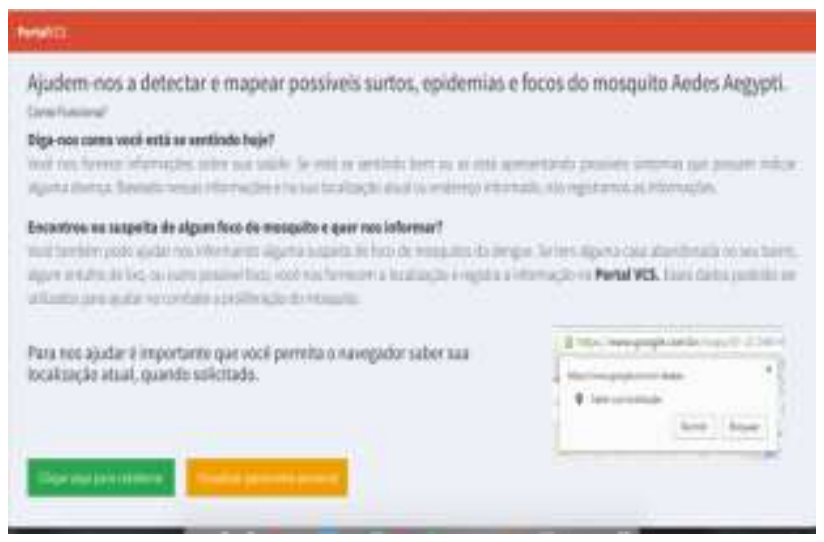

Figura 4: página inicial do PortalVCS

Ao escolher colaborar o usuário deverá efetuar o login com uma rede social de sua preferência, como mostra a Figura 5. O uso das redes sociais no login visa garantir a integridade das informações registradas no PortalVCS.

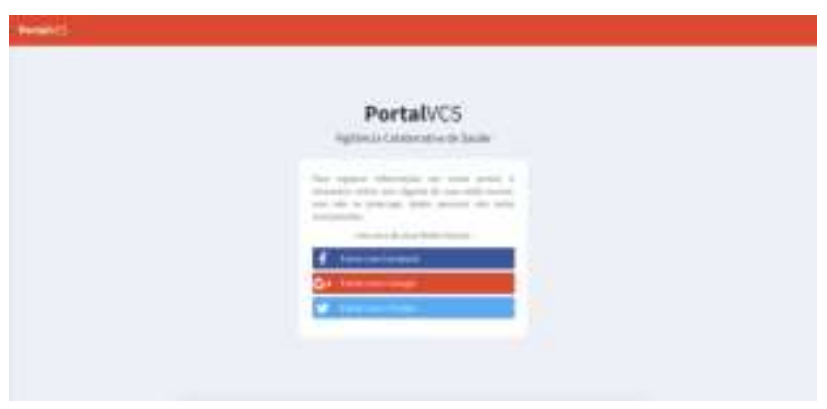

Figura 5: página de login com redes sociais.

Após o login o usuário e redirecionado para o primeiro passo que é onde ele seleciona o que deseja nos informar, se é como ele está se sentindo ou se é algum possível foco de mosquito da dengue. A Figura 6 mostra a tela na qual ele seleciona o que deseja informar.

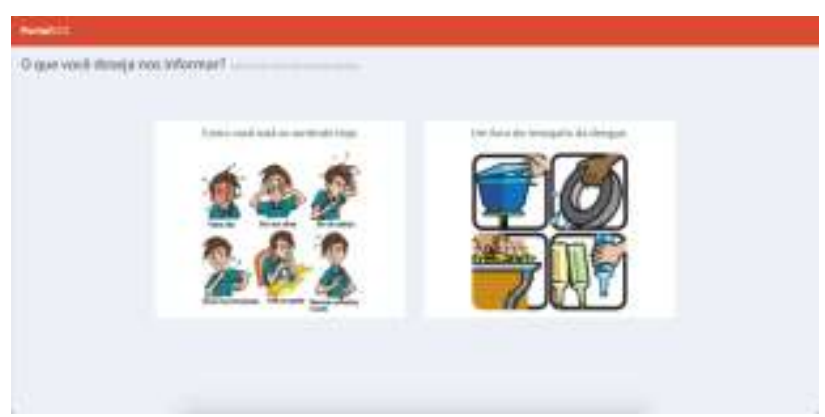

Figura 6: página na qual o usuário escolhe o que deseja informar. 
Caso o usuário escolha informar "como está se sentindo hoje" ele visualizará a página (Figuras 7,8 e 9) nas quais ele pode selecionar o que melhor representar sua situação atual de saúde. O mapa mostra sua localização atual, mas também é possível escolher um local diferente, caso ele deseje.

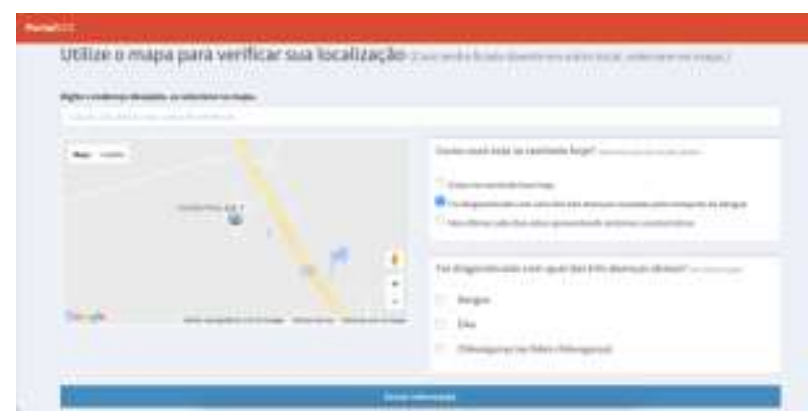

Figura 7: página em que o usuário informa como está se sentindo.

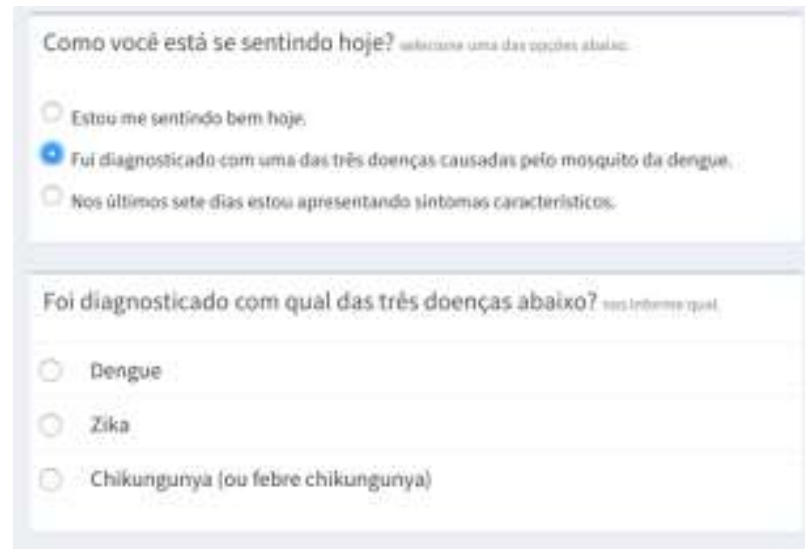

Figura 8: parte da tela que aparece caso o usuário selecione que foi diagnosticado com uma das 3 doenças causadas pelo mosquito.

No primeiro passo da aplicação o usuário também pode escolher informar um possível foco de proliferação de mosquito da dengue (Figura 6). Ao acessar ele irá para a página de registro. Assim como na página anterior (Figura 7) ele também visualizará um mapa onde informa sua localização atual, mas ele poderá informar uma localização diferente, afinal ele deverá marcar no mapa o local exato (ou mais próximo possível do foco que ele encontrou. A liberdade do usuário informar a localização exata do seu registro, também dá margem para que um usuário mal intencionado registre um dado falso, porém essa possibilidade deve ser sempre mantida para garantir a precisão do registro de um usuário que quer de fato colaborar. A tela possuirá também uma área de texto na qual ele pode escrever mais detalhes sobre o foco, caso deseje, como pode ser visto na Figura 10.

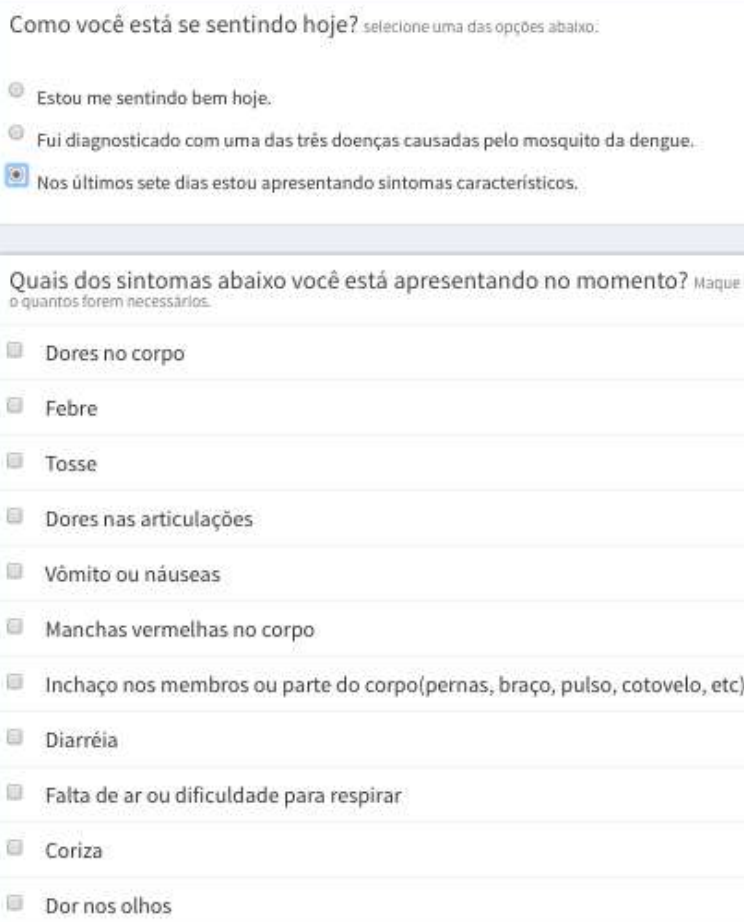

Figura 9: parte da tela que aparece caso o usuário selecione que está apresentando alguns sintomas.

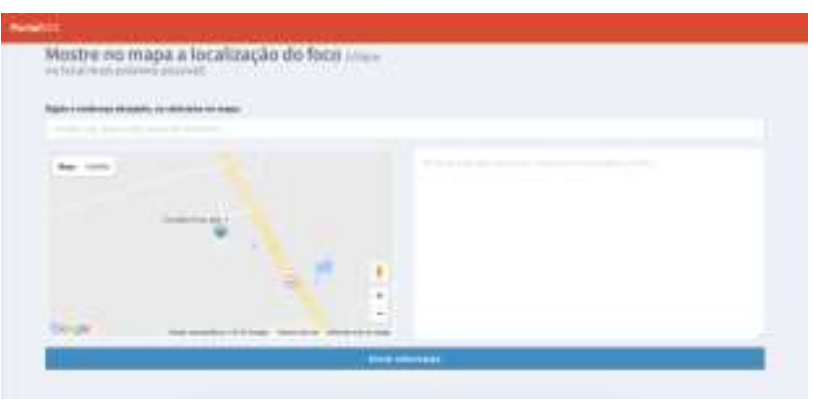

Figura 10: tela de registro de focos.

Ao final do registro de quaisquer informações o usuário sempre será redirecionado para a página inicial do PortalVCS (Figura 4), lá ele ainda tem mais uma opção que é o panorama semanal. Nessa opção o usuário informa uma cidade e em seguida ele visualizará no mapa todas as informações registradas naquela semana, verá também indicadores como total de registros, conforme mostra a Figura 11.

Os marcadores laranja com um ícone de alerta indicam focos de mosquito da dengue, marcadores verdes com um ícone feliz indica um registro de uma pessoa que está saudável e os marcadores vermelhos com um ícone triste são de registros que indicam ou algum diagnóstico de doença ou sintomas. 


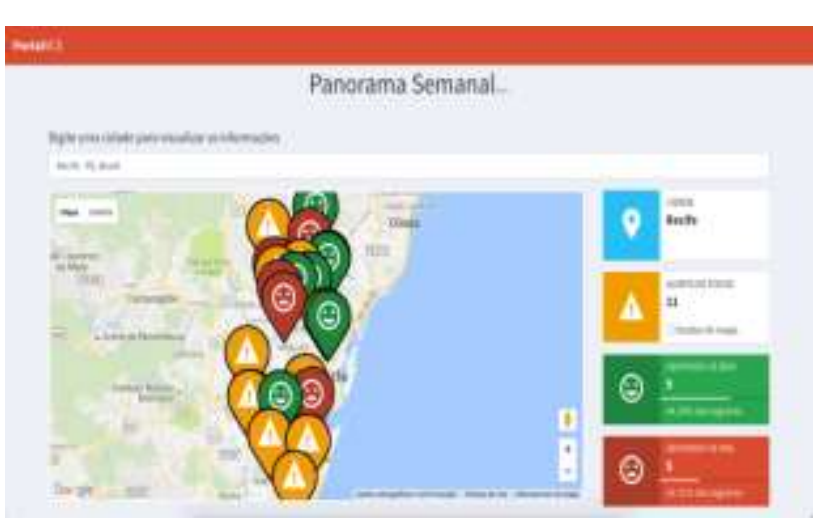

Figura 11: página de panorama semanal.

É possível interagir com o mapa, aumentando o zoom para ver a localização mais exata do marcador, como também é possível clicar nele para obter as informações enviadas pelo usuário. As Figuras 12, 13, 14 mostram como é feita a exibição das informações passadas pelo usuário.

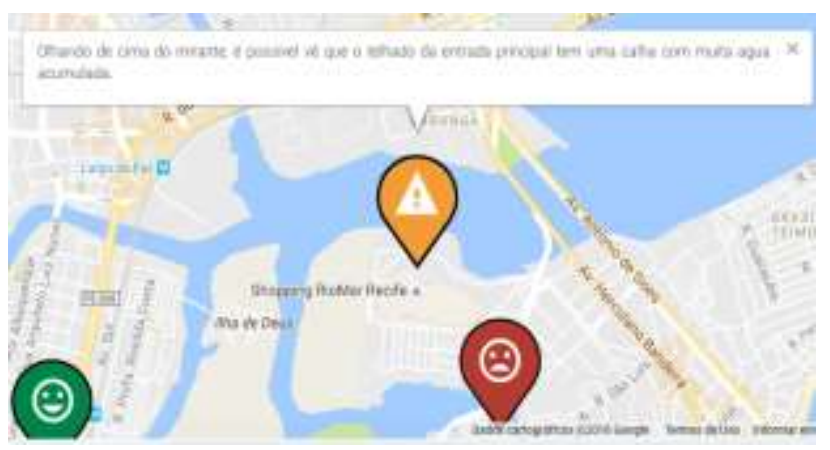

Figura 12: informação passada por um usuário em um registro de foco do mosquito da dengue.

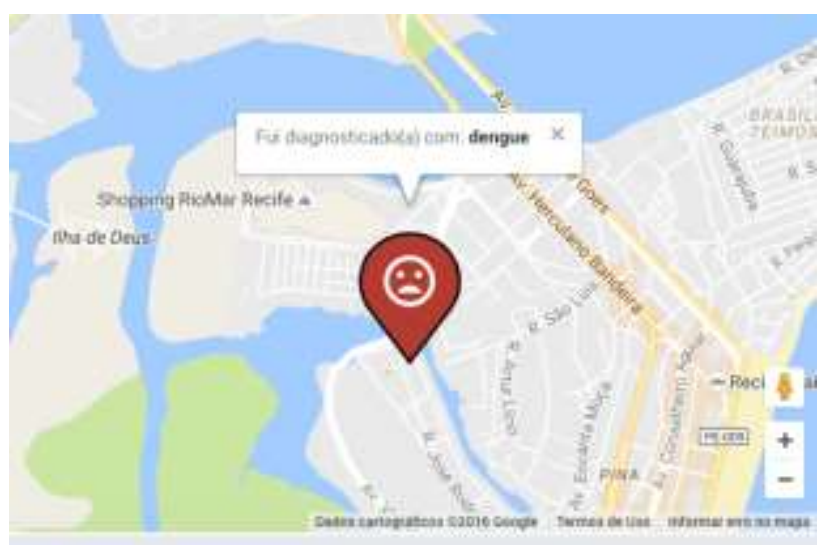

Figura 13: usuário informou que foi diagnosticado com dengue.

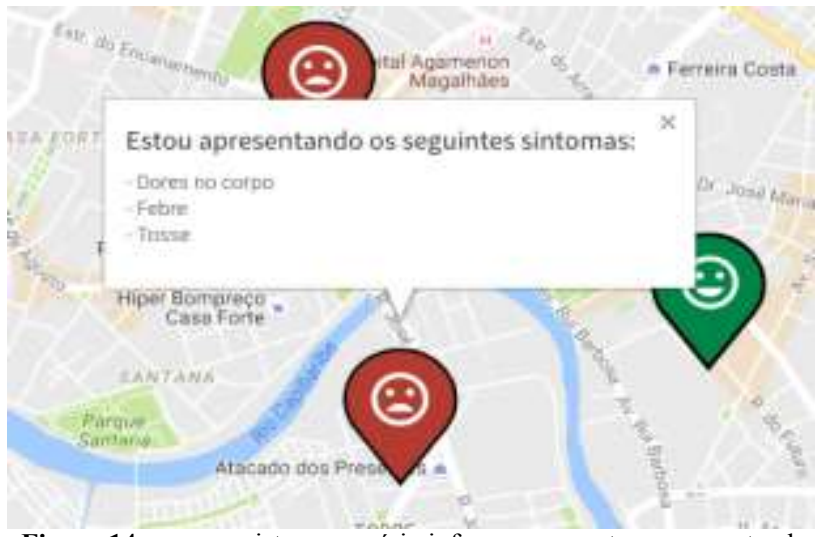

Figura 14: nesse registro, o usuário informou que estava apresentando alguns sintomas.

Todos os dados são registrados baseado nas coordenadas obtidas no mapa disponível para o usuário durante o registro da informação. Se o usuário não informar nenhum endereço ou ponto específico no mapa, a aplicação considerará a sua localização atual.

É possível fazer uma comparação entre o PortalVCS e alguns sistemas semelhantes mostrando as principais funcionalidades que eles atendem, conforme Tabela 1, onde uma tabela ajuda a visualizar melhor esta comparação.

\begin{tabular}{|c|c|c|c|c|c|c|}
\hline \multirow[b]{2}{*}{ SISTEMAS } & \multicolumn{6}{|c|}{ FUNCIONALIDADES } \\
\hline & $\begin{array}{c}\text { Colaboraçăo } \\
\text { direta do } \\
\text { usuario }\end{array}$ & $\begin{array}{c}\text { Colaboraçăo } \\
\text { Indireta do } \\
\text { usuário }\end{array}$ & $\begin{array}{l}\text { Registro de } \\
\text { Focos do } \\
\text { Mosquito da } \\
\text { Dengue }\end{array}$ & $\begin{array}{c}\text { Registro } \\
\text { informay̧ểes } \\
\text { sobre } \\
\text { sintomas }\end{array}$ & $\begin{array}{c}\text { Disponibiliza- } \\
\text { ço dos dados } \\
\text { para utilizaçao } \\
\text { pública }\end{array}$ & $\begin{array}{c}\text { Liberciade de } \\
\text { escolha da } \\
\text { localizaçăo } \\
\text { exata da } \\
\text { informaçăo } \\
\text { Registrada }\end{array}$ \\
\hline Guardioes da Saúde & SIM & NÃO & NÅO & SIM & SIM & NÃo \\
\hline Dengue na Web & SIM & NÅO & NÅO & SIM & NÃO & NÅO \\
\hline HealthMap & NÅ̃ & SIM & NÃO & NÃO & SIM & NÃO \\
\hline Observatório da Dengue & SIM & SIM & SIM & NÃO & NÅ̄O & NÃO \\
\hline PortalvCS & SIM & NÅO & SIM & SIM & SIM & SIM \\
\hline
\end{tabular}

Tabela 1: Comparação entre sistemas

\subsection{Tecnologias Utilizadas}

Desde a concepção do PortalVCS até o seu desenvolvimento foi levado em conta o uso de tecnologias atuais, fácil manutenção, alta produtividade e baixo custo. A Figura 15 ajuda a entender melhor como foi utilizada as tecnologias no desenvolvimento do PortalVCS.

$\mathrm{Na}$ arquitetura do projeto, foi utilizado o padrão MVC. Para o estilo e apresentação foi utilizado o framework responsivo Bootstrap que é bastante conhecido e utilizado por desenvolvedores Web. No client-side foi utilizado os três pilares do desenvolvimento Web: HTML5, CSS3 e JavaScript, mas para toda a parte de lógica, gerenciamento de navegação entre páginas, comunicação com o servidor, foi utilizado o framework JavaScript AngularJS. 


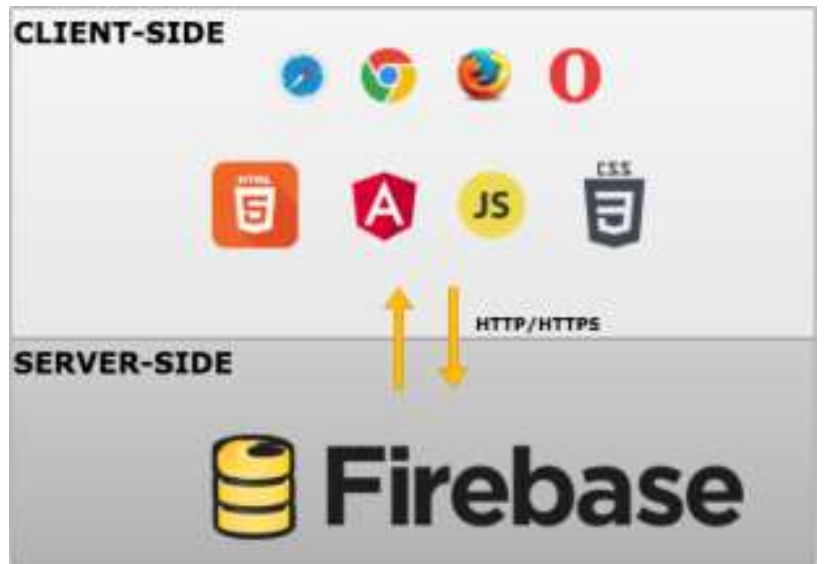

Figura 15: arquitetura da aplicação.

O AngularJS é um Framework estrutural para o desenvolvimento de aplicações Web. Foi desenvolvido pela Google com o objetivo de trazer para o lado do cliente ferramentas e funcionalidades que geralmente são utilizadas no nível do servidor. Com isso, espera-se facilitar o desenvolvimento, testes e manutenção dos sistemas. É um arquivo JavaScript, que não precisa ser instalado, apenas importado em sua aplicação (ANGULARJS).

O AngularJS faz o uso de conceitos como Data Binding e injeção de dependências. O Data Binding é uma técnica que se constitui em mudar algo no modelo da aplicação e refletir no DOM essas alterações, sem a necessidade de recarregar a página. Isso significa dizer que é possível alterar apenas uma parte da página ou template, sem ter que recarregá-la completamente, porém essa técnica demanda cuidados e conhecimentos mais avançados que acaba não sendo tão simples de se utilizar. No AngularJS o data binding acontece de forma automática minimizando esse tipo de preocupação. Nele é implementado um tipo de data binding chamado de twoway data binding que consiste em modificar o DOM e refletir nos objetos ou modificar o objeto e espelhar a modificação no DOM.

Injeção de dependência é um conjunto de princípios e padrões que permitem o desenvolvimento de aplicações menos acopladas, através de inclusão de bibliotecas e/ou dependências em um módulo quando este for necessário.

Para a parte de mapas e endereços foi utilizado a API de mapas do Google, mas por meio de uma diretiva AngularJS chamada NG-MAP, que facilita a implementação e o uso de todas as funcionalidades oferecidas pela Google.

No lado do servidor (server-side), foi utilizado um único o Firebase fornecido pela empresa também de mesmo nome. Esta foi Fundada em 2011 por Andrew Lee e James Tamplin e em 2014 foi comprada pela Google.

O Firebase é um BaaS (backend as service) que for- nece vários serviços para facilitar o desenvolvimento de aplicações no lado do servidor. Tem integração a várias plataformas como AngularJS, Node.js, JavaScript, Android e IOS.

O sistema de armazenamento de dados do Firebase é um banco NoSQL realtime que armazena os dados na nuvem e toda a manipulação desses dados é feito por meio de uma API REST, mas que todas as chamadas podem ser feitas através das bibliotecas específicas de cada linguagem.

O Firebase reduz a complexidade e até elimina a necessidade de desenvolver uma aplicação server-side específica. É um serviço pago que possui um plano free com algumas limitações, mas que atendeu perfeitamente todas as necessidades para o desenvolvimento desse trabalho.

\section{Resultados Obtidos}

Durante os dias 25 de julho de 2016 ao dia 05 de agosto de 2016 o PortalVCS foi liberado para a coleta de dados. Nesse período houve um total de 46 registro de informações em três cidades de Pernambuco que foram Recife, Olinda e São Lourenço da Mata e uma cidade no Ceará que foi a capital Fortaleza.

Dos 46 registros de informações desse período, 22 deles foram referentes a possíveis focos de mosquito da dengue, isso equivale a $47,83 \%$ do total, e $52,17 \%$ foram de registros onde pessoas informaram como estavam se sentindo. A Figura 16 mostra um gráfico por cidades.

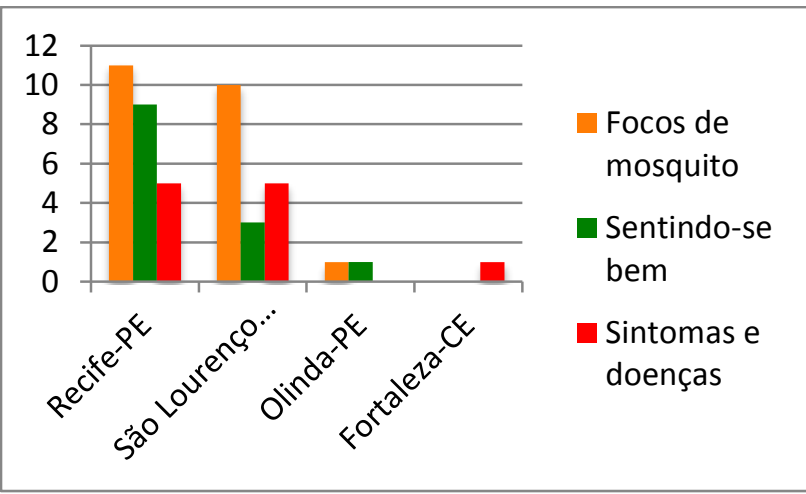

Figura 16: Gráfico com a distribuição dos registros por cidade: Recife com um total de 25 registros, São Lourenço da Mata com 18 registros, Olinda com 2 registros e Fortaleza - CE com 1 registro.

De modo geral os usuários reportaram que acharam a ideia pertinente, a ferramenta útil e de fácil manuseio. Foi observado que na página de registro de focos de mosquito, a área destinada ao usuário escrever mais detalhes, foi muito bem aproveitada, pois os registros continham informações claras que facilitava a detecção do local que ele estava informando, um exemplo disso pode ser observado na Figura 10. 
Outro ponto analisado foi que alguns usuários, utilizaram o PortalVCS mais de uma vez. Em virtude dos benefícios oferecidos pela API de mapas do Google é possível interagir de diversas formas com o mapa, sendo possível alcançar um nível de visualização bastante detalhado, como por exemplo, usar as imagens de satélite para tentar localizar o local exato informado por um usuário. O AngularJS se mostrou bastante poderoso e versátil. Com ele foi possível implementar algumas regras de negócio que rodam do lado do cliente sem atrapalhar a performance e experiência de acesso da aplicação.

O Firebase foi importante no desenvolvimento do PortalVCS, pois ele abstraiu totalmente a necessidade do desenvolvimento server-side. Toda parte de armazenamento dos dados, gerenciamento de login do usuário e hospedagem da aplicação foi feita utilizando as ferramentas oferecidas por ele.

\section{Conclusões e Trabalhos Futuros}

O objetivo deste artigo foi propor uma ferramenta capaz de auxiliar o combate as epidemias e surtos das doenças causadas pelo mosquito Aedes Aegypit bem como sua proliferação, utilizando os benefícios e os avanços das tecnologias da informação e comunicação, como também a participação ativa da população (crowdsourcing ativo). A ferramenta funciona como um aliado ao trabalho dos agentes de saúde e endemias que vão nos bairros em procura de focos e pessoas doente, de forma que essa tarefa seja feita forma muito mais eficiente.

A partir dos testes realizados, foi observado o quão eficiente e útil uma ferramenta deste tipo pode ser, já que existe um grande nível de detalhes nas informações lá contidas, como por exemplo a localização exata de um possível foco do mosquito. Os testes serviram também para obter um feedback dos usuários no sentido de prover melhorias e identificar possíveis ajustes. Foi possível ainda perceber que a colaboração dos usuários é imprescindível para o funcionamento pleno da ferramenta, visto que na versão atual as informações advém unicamente dos usuários.

Durante os testes dos usuários houveram várias sugestões de melhorias dentre pode-se destacar: a implementação de um sistema de gamificação onde o usuário seja estimulado a sempre colaborar com o sistema. Transformar o PortalVCS em um aplicativo totalmente mobile, na qual fosse possível baixá-lo nas lojas de aplicativos como Apple Store e Google Play, etc. Todas as sugestões ficarão para melhorias futuras da aplicação.

Também foi observado a importância de implementar uma funcionalidade que extraia informações de forma autônoma da Web através da mineração de dados em redes sociais e sites específicos da área (crowdsourcing passivo). Essa melhoria permitiria que o sistema não dependesse exclusivamente da participação do usuário.

O trabalho tenta deixar clara a importância do uso de plataformas de vigilância colaborativa como um aliado no combate a epidemias e surtos, uma vez que a ideia principal deste trabalho pode ser utilizada como base para o combate de várias outras doenças

\section{Referências}

[1] ANGULARJS. What is Angular?: https://docs.angularjs.org/guide/introduction acessado em: 30/07/2016.

[2] BATURAY, Meltem Huri; BIRTANE, Murat. Responsive web design: a new type of design for web-based institucional contente. ProcediaSocial and Behavioral Sciences, v.106, p. 22752279, 2013.

[3] DAL MORO, Tharcis; DORNELES, Carina; REBONATTO, Marcelo Trindade. Web services WS-* versus Web Services REST. Revista de Iniciação Científica, v. 11, n. 1, 2009.

[4] DA SILVA BARROS, Daniele Montenegro et al. OBSERVATÓRIO NACIONAL DA DENGUE-SISTEMA PARA MONITORAMENTO DE CASOS DE DENGUE. Revista Brasileira de Inovação Tecnológica em Saúde ISSN: 2236-1103, v. 3, n. 4, 2014.

[5] DE CASTRO ZORZO, Rafael; BERNARDI, Élder FF. Estudo e Desenvolvimento de Caso De Uso com Framework Angularjs.

[6]

DENGUE NA WEB: https://www.denguenaweb.ufba.br acessado em 25/05/2016.

[7] FLU NEAR YOU: https://www.flunearyou.org/ acessado em: 25/05/2016.

[8] FREIFELD, Clark C. et al. HealthMap: global infectious disease monitoring through automated classification and visualization of Internet media reports.Journal of the American Medical Informatics Association, v. 15, n. 2, p. 150-157, 2008.

[9] Guardiões da Saúde link: https://guardioesdasaude.org/ Acessado em: 07/07/2016.

[10] GOMES, Carolina B. et al. O novo Regulamento Sanitário Internacional. Revista de Direito Sanitário, v. 13, n. 2, p. 137-155, 2012.

[11] HANSEN, Roseli Persson et al. Web services: an architectural overview. In:First Internation- 
al Seminar on Advanced Research in EBusiness-EBR. 2002.

[12] HARRISON, Neil B.; AVGERIOU, Paris; ZDUN, Uwe. Using patterns to capture architectural decisions. IEEE software, v. 24, n. 4, p. 38-45, 2007.

[13] INFLUENZANET: https://www.influenzanet.eu/ acessado em: 20/05/2016.

[14] LEAL-NETO, Onicio B et al . Detecção digital de doenças e vigilância participativa: panorama e perspectivas para o Brasil. Rev. Saúde Pública, São Paulo, v. 50, 17, 2016.

[15] LOWE, David. Web system requirements: an overview. Requirements Engineering, v. 8, n. 2, p. 102-113, 2003.

[16] MINISTÉRIO DA SAÚDE DO BRASIL, Boletim Epidemiológico - Secretaria de Vigilância em Saúde - Volume $47 N^{\circ}$ 20, 2016.

[17] MORO VISCONTI, Roberto; LAROCCA, Alberto; MARCONI, Michele. Malaria GeoLocalization with Mobile-Health Applications. Available at SSRN, 2015.

[18] MORSE, Stephen S. et al. ProMED global monitoring of emerging diseases: design for a demonstration program. Health Policy, v. 38, n. 3, p. 135-153, 1996.

[19] MYKHALOVSKIY, Eric; WEIR, Lorna. The Global Public Health Intelligence Network and early warning outbreak detection: a Canadian contribution to global public health. Canadian Journal of Public Health/Revue Canadienne de Sante'e Publique, p. 42-44, 2006 